\title{
Metastasis-associated in colon cancer-1 is associated with poor prognosis in hepatocellular carcinoma, partly by promoting proliferation through enhanced glucose metabolism
}

\author{
YAOQING LI, ZHONGTANG LU, ZHEYONG LIANG, DONG JI, PENGFEI ZHANG, \\ QINGGUANG LIU, XIN ZHENG and YINGMIN YAO \\ Department of Hepatobiliary Surgery, The First Affiliated Hospital of the Medical College of Xi'an Jiaotong University, \\ Xi'an, Shaanxi 710061, P.R. China
}

Received March 1, 2014; Accepted January 21, 2015

DOI: $10.3892 / \mathrm{mmr} .2015 .3416$

\begin{abstract}
Metastasis-associated in colon cancer-1 (MACC1) is a newly identified gene that is involved in the development and progression of hepatocellular carcinoma (HCC), however its investigation has not been comprehensive. In the present study, in vitro techniques, including immunohistochemistry, western blotting, reverse transcription quantitative polymerase chain reaction, metabolic assay, MTT assay, colony formation assay and prognostic analysis were used to confirm the involvement of MACC1 in HCC. Histological examination confirmed that the protein expression of MACC1 was upregulated in HCC and was associated with the hexokinase-2 (HK2) protein, which also indicates a poor prognosis. Knockdown of MACC1 induced the reduction of glycogen consumption and lactate production, which then lead to a marked reduction of proliferation in the MHCC-97H cells. However, the overexpression of MACC1 produced the opposite results in the HepG2 cells. These results suggested that MACC1 leads to a poor prognosis in HCC, partly by promoting proliferation via enhancement in glucose metabolism by HK2. Therefore, this pathway has the potential to become an important therapeutic target in HCC.
\end{abstract}

Correspondence to: Mr. Yingmin Yao, Department of Hepatobiliary Surgery, The First Affiliated Hospital of the Medical College of Xi'an Jiaotong University, 277 Yanta West Road, Xi'an, Shaanxi 710061, P.R. China

E-mail: yaoyingmin@sina.com

Abbreviations: MACC1, metastasis-associated in colon cancer-1; HK-2, hexokinase-2; HCC, hepatocellular carcinoma; OS, overall survival CI, confidential intervals; HR, hazard ratio

Key words: metastasis-associated in colon cancer-1, hexokinase-2, glucose metabolism, proliferation, hepatocellular carcinoma, prognosis

\section{Introduction}

Hepatocellular carcinoma (HCC) is the fifth most common type of cancer and has the third highest rate of tumor-associated mortality worldwide (1). Surgical resection is one of the most effective treatment options for patients with HCC (2). However, the majority of patients are unable to undergo curative resection due to the advanced cancer staging when first diagnosed. This is considered to be associated with the distinctive rapid progression observed in HCC. Therefore, there is an urgent requirement to understand the mechanism underlying the rapid progression of HCC.

Metastasis-associated in colon cancer-1 (MACC1) is a novel oncogenic factor involved in tumor growth and metastasis, and is aberrantly overexpressed in various types of tumor, including lung cancer (3), gastric cancer (4), colorectal cancer $(5,6)$ and breast cancer (7). Our preliminary investigation revealed that MACC1 was aberrantly upregulated in HCC tissues compared with adjacent liver tissues, and in vitro experiments revealed that the overexpression of MACC1 promoted the migration and invasive ability of HCC cells, potentially via upregulating matrix metalloproteinase (MMP)2 and MMP9 (8). Another study demonstrated that overexpression of MACC1 induces the increasing expression of downstream factors, including hepatocyte growth factor (HGF) and C-met, and promotes tumor growth and metastasis in colorectal cancer $(9,10)$. However, the molecular mechanism whereby MACC1 leads to HCC progression remains to be elucidated.

In the 1930s, Warburg et al described the phenomenon that glycolysis was increased despite a adequate supply of oxygen, which is now well-known as the Warburg effect in cancer cells (11). Hexokinase-2 (HK2), identified as a key rate-limiting enzyme in glucose metabolism, catalyzes the reaction of the first step of glycolysis and has an irreplaceable role in cancer glucose metabolism (12). Furthermore, with the unlimited proliferation of tumor cells, the demand for energy and the expression of basic glycolytic enzymes, particularly HK2, increases markedly in the majority of tumors $(13,14)$. In addition, the overexpression of HK2 enhances the affinity of tumor cells for adenosine triphosphate (ATP), promotes tumor cells to uptake ATP and, in turn, improves the level 
of oxidative phosphorylation in an environment of low ATP (15).

The MACC1-HGF/C-met pathway is important in cellular growth, epithelial-mesenchymal transition, angiogenesis, cell motility, invasiveness and metastasis through the activation of the mitogen-activated protein kinase (MAPK) and phosphoinositide 3-kinase (PI3K)-Akt signaling pathways $(9,16,17)$. To assess the correlation between MACCl and cancer glucose metabolism in HCC, the present study used in vitro assays to investigate the expression of $\mathrm{HK} 2$, an essential glycolytic enzyme and downstream factor of the PI3K/AKT signaling pathway $(18,19)$, and analyzed the correlation between the two proteins and their role in postsurgical survival.

\section{Materials and methods}

Patients and specimens. A total of 80 patients with HCC [Child-Pugh A to B, scored as previously described (20)] were registered in the present study between February 2006 and January 2008, including 58 males and 22 females (mean age 51 years; range 24-76), who had not received preoperative chemotherapy or embolization. Following necessary preoperative examinations, all the patients underwent liver resection. Tumor tissue and matched normal tumor-adjacent tissue specimens ( $\geq 2 \mathrm{~cm}$ distance to the resection margin) were collected and immediately stored in paraformaldehyde for immunohistochemical analysis or at $-80^{\circ} \mathrm{C}$ for western blot analysis, respectively. Clinical data were obtained from the patient's medical records.

Written informed consent was obtained from all patients. The Xi'an Jiaotong University Ethics committee approved all procedures, according to the The Declaration of Helsinki, 1975 (21).

Cell culture and transfection. Liver cell lines (HepG2, Hep3B, SMMC-7721, Bel-7402, Huh7, MHCC-97H and LO2) were obtained from the Institute of Biochemistry and Cell Biology, Chinese Academy of Sciences (Shanghai, China). All the cells were maintained in Dulbecco's modified Eagle's medium (DMEM; Hyclone, Logan, UT, USA) containing 10\% fetal bovine serum (FBS; Gibco BRL, Gaithersburg, MD, USA) with $100 \mathrm{U} / \mathrm{ml}$ penicillin and $100 \mu \mathrm{g} / \mathrm{ml}$ streptomycin (Sigma-Aldrich, St. Louis, MO, USA) and cultured in a humidified $5 \% \mathrm{CO}_{2}$ incubator at $37^{\circ} \mathrm{C}$ for $48-72 \mathrm{~h}$.

The MACC1-expressing plasmid and control plasmid (pCMV6-Entry; GenePharma, Shanghai, China) were transfected into HepG 2 cells using Roche FuGENE ${ }^{\circledR} 6$ Transfection reagent (Roche Diagnostics, Indianapolis, IN, USA), and stably expressing clones were selected by G418 at a dose of $300 \mu \mathrm{g} / \mathrm{ml}$ for 2 weeks. Western blot analysis confirmed the overexpression of MACC1 in the selected stably expressing clones (Fig. 5A). MACC1 small interfering (si)RNA and control siRNA were transfected into the MHCC-97H cells using the siPORT ${ }^{\mathrm{TM}} \mathrm{NeoFX}^{\mathrm{TM}}$ transfection agent purchased from Applied Biosystems (Carlsbad, CA, USA).

Immunohistochemistry. Tumor samples were fixed in $10 \%$ buffered formalin solution (Shaanxi Xianfeng Biotechnology Co., Ltd, Xi'an, China) and embedded in paraffin (Shaanxi Xianfeng Biotechnology Co., Ltd). Rabbit polyclonal MACC1 (cat. no. ab106579; Abcam, Hong Kong, China;1:200) and rabbit monoclonal HK2 (cat. no. C64G5; Cell Signaling Technology, Danvers, MA, USA; 1:100) antibodies were used in immunohistochemical analysis (IHC) using the streptavidin-peroxidase conjugated method. In brief, following antigen retrieval in a citrate buffer (Shaanxi Xianfeng Biotechnology Co., Ltd) using a microwave (350W; Midea Group Co., Ltd, Guangdong, China) for $15 \mathrm{~min}$; the sections were then incubated with normal serum (Shaanxi Xianfeng Biotechnology Co., Ltd) at $37^{\circ} \mathrm{C}$ for $30 \mathrm{~min}$ and then with the primary antibody against MACC1 or $\mathrm{HK} 2$ at $4^{\circ} \mathrm{C}$ overnight. Sections were washed with PBS (Shaanxi Xianfeng Biotechnology Co., Ltd) and incubated with biotinylated goat anti-rabbit monoclonal secondary antibodies (cat no. SP-9000-D; Zhongshan Goldenbridge Biotechnology Co., Ltd, Beijing, China) and avidin-biotin-peroxidase complex (Zhongshan Goldenbridge Biotechnology Co., Ltd) according to the manufacturer's instructions. The positive signal was visualized by incubating the sections with a diaminobenzidine solution (Shaanxi Xianfeng Biotechnology Co., Ltd) and counterstaining with hematoxylin (Shaanxi Xianfeng Biotechnology Co., Ltd). The sections were then observed under an inverted microscope (Nikon China Co., Ltd, Tokyo, Japan). The staining intensity was expressed as four grades: 0 , none; 1, weak; 2, moderate; and 3 , strong under 10 randomly selected independent high magnification (magnification, x400) fields. The percentages of positive carcinoma cells were expressed as the following grades: $0,<10 \% ; 1,10-25 \% ; 2,26-50 \% ; 3,51-75 \%$; and $4,>75 \%$. The total score was calculated by summating the staining intensity and the percentage of positive tumor cells. Sections with a total score $>2$ were defined as exhibiting positive staining for the above two proteins.

Western blot analysis. Rabbit polyclonal MACC1 (cat. no. ab106579; Abcam; 1:1,000), rabbit monoclonal HK2 $(1: 1,000)$ and mouse monoclonal $\beta$-actin (cat. no. sc-47778, Santa Cruz Biotechnology, Inc., Santa Cruz, CA, USA; 1:1,000) antibodies were used for western blot analysis. Secondary horseradish peroxidase-conjugated goat anti-mouse or rabbit antibodies (Bio-Rad, Laboratories, Inc., Hercules, CA, USA) were used at a 1:5,000 dilution.

Briefly, equal quantities of the protein samples were separated by denaturing gel electrophoresis (Bio-Rad Laboratories, Inc.). Following transfer onto a polyvinylidene difluoride membrane (Millipore, Billerica, MA, USA) blots were probed overnight with the primary antibodies (MACC1, HK2 and $\beta$-actin) respectively. Following washing 3 times in Tris-buffered saline with Tween 20 (Sino-American Biotechnology, He'nan, China), blots were then incubated with the relevant goat anti-rabbit and goat anti-mouse immunoglobulin $\mathrm{G}$ monoclonal secondary antibodies (cat nos. sc-2004 and sc-2005; Santa Cruz Biotechnology, Inc.; 1:15,000) conjugated with horseradish peroxidase (HRP) and signals were visualized using the HyGLO HRP detection kit from Denville Scientific (Metuchen, NJ, USA).

Reverse transcription quantitative polymerase chain reaction $(R T-q P C R)$. Total cellular RNA was extracted from the cultured cells using a Fastgen200 RNA isolation System (Fastgen, Shanghai, China). cDNA synthesis was achieved using the High Capacity cDNA Reverse Transcription kit 
Table I. Distribution of MACC1 and HK2 positive tumors in groups, defined by clinicopathological variables.

\begin{tabular}{|c|c|c|c|c|c|c|c|}
\hline \multirow[b]{2}{*}{ Variable } & \multirow[b]{2}{*}{ Group } & \multicolumn{2}{|c|}{ MACC1 } & \multirow[b]{2}{*}{ P-value } & \multicolumn{2}{|c|}{ HK2 } & \multirow[b]{2}{*}{ P-value } \\
\hline & & + & - & & + & - & \\
\hline \multirow[t]{2}{*}{ Age } & $>50$ & 32 & 12 & 0.818 & 33 & 11 & 0.281 \\
\hline & $\leq 50$ & 27 & 9 & & 23 & 13 & \\
\hline \multirow[t]{2}{*}{ Gender } & Male & 41 & 17 & 0.312 & 40 & 18 & 0.743 \\
\hline & Female & 18 & 4 & & 16 & 6 & \\
\hline \multirow[t]{2}{*}{ HBV infection } & Yes & 47 & 15 & 0.438 & 44 & 18 & 0.726 \\
\hline & No & 12 & 6 & & 12 & 6 & \\
\hline \multirow[t]{2}{*}{ Liver cirrhosis } & Yes & 43 & 14 & 0.589 & 40 & 17 & 0.957 \\
\hline & No & 16 & 7 & & 16 & 7 & \\
\hline \multirow[t]{3}{*}{$\mathrm{AFP}(\mathrm{ng} / \mathrm{ml})$} & $>20$ & 42 & 12 & 0.445 & 37 & 17 & 0.913 \\
\hline & $\leq 20$ & 14 & 8 & & 16 & 6 & \\
\hline & Unknown & 3 & 1 & & 3 & 1 & \\
\hline \multirow[t]{3}{*}{ Tumor size $(\mathrm{cm})$} & $\geq 5$ & 36 & 4 & $0.004^{\mathrm{a}}$ & 27 & 12 & 0.738 \\
\hline & $<5$ & 21 & 16 & & 28 & 10 & \\
\hline & Unknown & 2 & 1 & & 1 & 2 & \\
\hline \multirow[t]{2}{*}{ Edmondson } & $\mathrm{I}+\mathrm{II}$ & 30 & 17 & $0.016^{\mathrm{a}}$ & 26 & 21 & $0.001^{\mathrm{a}}$ \\
\hline & III+IV & 29 & 4 & & 30 & 3 & \\
\hline \multirow[t]{2}{*}{ TNM stage } & $\mathrm{T} 1+\mathrm{T} 2$ & 28 & 16 & $0.023^{\mathrm{a}}$ & 24 & 20 & $0.001^{\mathrm{a}}$ \\
\hline & $\mathrm{T} 3+\mathrm{T} 4$ & 31 & 5 & & 32 & 4 & \\
\hline Intrahepatic & Yes & 12 & 1 & 0.097 & 11 & 2 & 0.209 \\
\hline metastases & No & 47 & 20 & & 45 & 22 & \\
\hline Portal vein & Yes & 11 & 1 & 0.126 & 9 & 3 & 0.682 \\
\hline Invasion & No & 48 & 20 & & 47 & 21 & \\
\hline
\end{tabular}

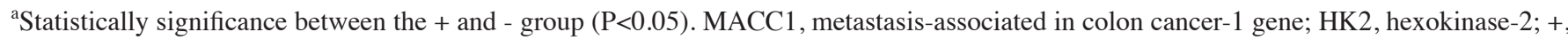
positive tumors; -, negative tumors. HBV, hepatitis B virus; AFP, serum $\alpha$-fetoprotein; TNM, tumor-node-metastasis.

(Fermentas, Burlington, Canada). RT-qPCR was performed using SYBR Green PCR master mix (Takara Bio, Inc., Dalian, China) using an IQ5 system (Bio-Rad Laboratories, Inc.). The primers to detect mRNA were as follows: MACC1, forward 5'-CTTGCGGAGGTCACCATAGC-3' and reverse 5'-GATTTCCAACAACGGGCTCA-3'. All the samples were normalized to internal controls and fold-changes were calculated through relative quantification. Each measurement was performed in triplicate.

Metabolic assay of glycogen. Glycogen measurements were performed using a glycogen concentration kit (Biovision, Milpitas, CA, USA) according to the $\mathrm{KOH}$-anthrone method, described by Liu et al (22). Lactate quantifications in the media, following transfection or planting, were performed using a Lactate assay kit II (Biovision). Experiments were performed in triplicate.

MTT assay. Proliferation was determined using an MTT assay (Roche Diagnostics). Following transfection of the MACC1 siRNA or MACC1 expressing plasmids, the cells were cultured further for $10-20 \%$ area of a culture dish, in a humidified $5 \% \mathrm{CO}_{2}$ incubator at $37^{\circ} \mathrm{C}$ for 0 to $72 \mathrm{~h}$ and the absorbance of the samples was measured using a model 550 microplate reader (Bio-Rad
Laboratories, Inc.), at a wavelength of $570 \mathrm{~nm}$ corrected to $655 \mathrm{~nm}$. The experiments were performed in triplicate.

Colony formation assay. A total of 100 HepG2-vector and HepG2-MACC1 cells were placed in a fresh six-well plate in triplicate and maintained in DMEM containing 10\% FBS in a humidified $5 \% \mathrm{CO}_{2}$ incubator at $37^{\circ} \mathrm{C}$ for 2 weeks. The cell colonies were fixed with $20 \%$ methanol and stained with $0.1 \%$ coomassie brilliant blue R250 at room temperature for $15 \mathrm{~min}$. The colonies were counted using a ELIspot Bioreader 5000 (Bio-Sys, Karben, Germany).

Statistical analysis. Differences between groups were compared using the $\chi^{2}$ test, Fisher's exact test, the Mann-Whitney test and an analysis of variance. $\mathrm{P}<0.05$ was considered to indicate a statistically significant difference. The SPSS 13.0 statistical package (SPSS, Inc., Chicago, IL, USA) was used for all calculations.

\section{Results}

MACC1 is overexpressed and accompanied by the expression of HK2 in HCC tissue. The IHC staining of the protein expression of MACC1 was positive in 59/80 (73.8\%) of the 

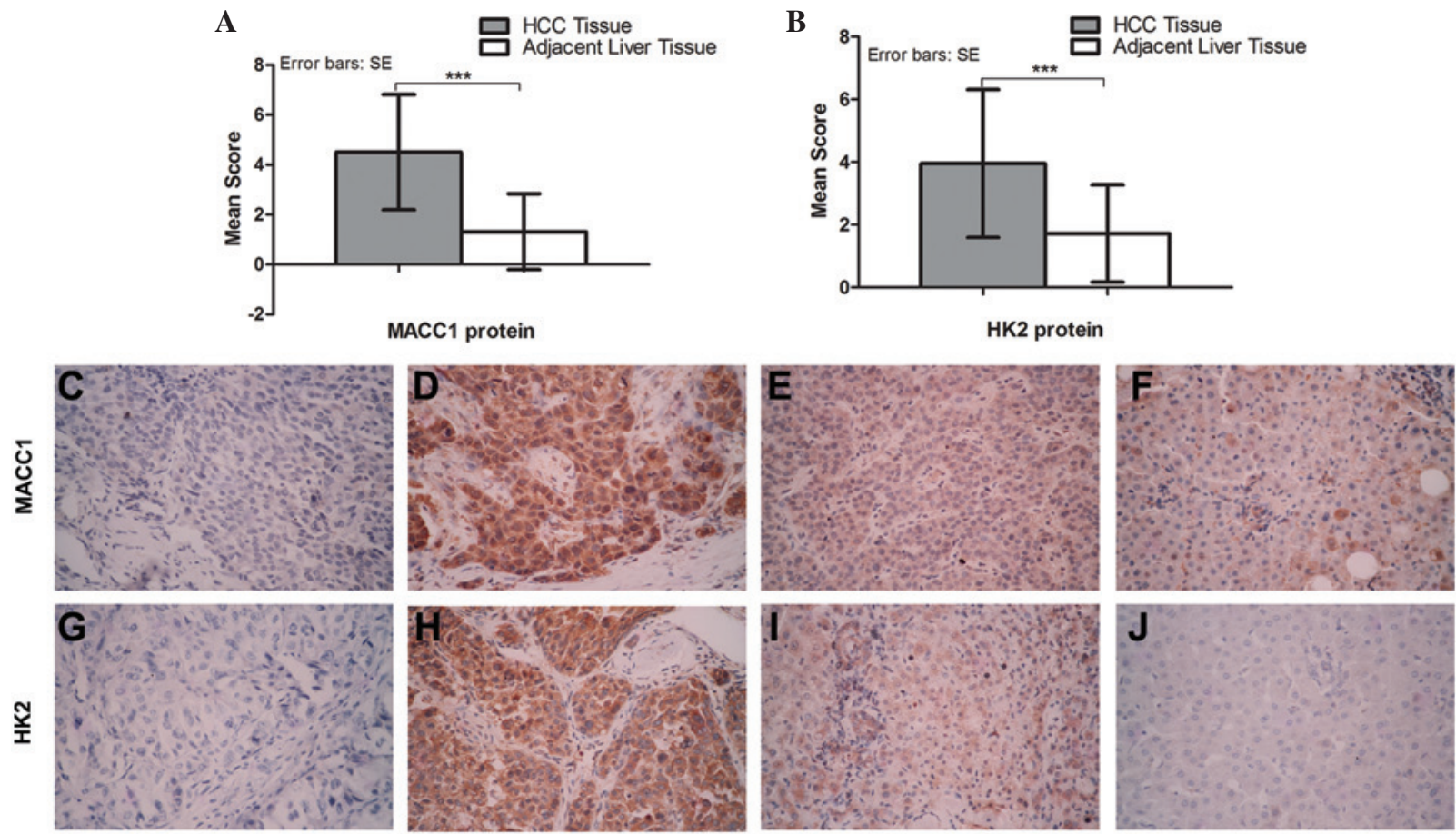

HCC Tissue

HCC Tissue

HCC Tissue

Adjacent Liver Tissue

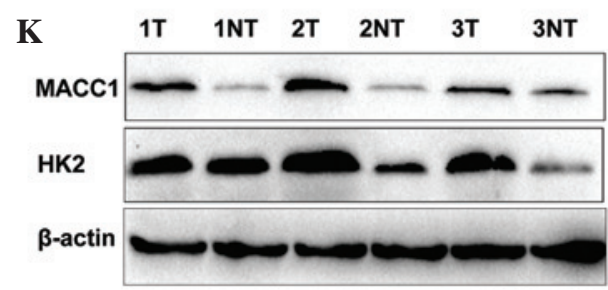

Figure 1. Immunohistochemical staining and western blotting of MACC1 and HK2 in HCC tissues and adjacent liver tissues. (A) Protein expression of MACC1 in HCC tissues and matched adjacent liver tissues $(\mathrm{n}=80$ ). (B) Protein expression of HK2 in HCC tissues and matched adjacent liver tissues ( $\mathrm{n}=80$ ). Values are expressed as the mean \pm standard error of the mean comparing the HCC with the adjacent liver tissues $\left({ }^{* * *} \mathrm{P}<0.001\right)$. (C and $\left.\mathrm{G}\right)$ Negative control in $\mathrm{HCC}$ tissues. (D and H) High intensity tumor staining for MACC1 and HK2 from the same patient, respectively. (F and J) Corresponding adjacent liver tissues exhibited weak expression of MACC1 and no expression of HK2. (E and I) Moderate expression levels of MACC1 and HK2 were observed in the HCC tissues (magnification, x400). (K) Protein expression levels of MACC1 and HK2 in HCC tissues (T) and adjacent non-tumorous liver tissues (NT). Numbers 1-3 indicate different tissue samples. HK2, hexokinase-2; MACC1, metastasis-associated in colon cancer-1; HCC, hepatocellular carcinoma.

HCC tissues compared with 23/80 (28.8\%) of the adjacent liver tissues, while the protein expression of HK2 was positive in 56/80 (70\%) and 25/80 (31.3\%; $\mathrm{P}<0.001)$, respectively. In addition, the IHC scores also demonstrated that the protein expression of MACC1 was significantly higher in the HCC tissues compared with the adjacent liver tissues (4.50 \pm 2.31 , vs. $1.32 \pm 1.52 ; \mathrm{P}<0.001 ;$ Fig. $1 \mathrm{~A})$, as was the protein expression of HK2 (3.95 \pm 2.36 vs. $1.72 \pm 1.55 ; \mathrm{P}<0.001$; Fig. 1B). The results of the IHC assay demonstrated that MACC1 protein was located predominantly in the nucleus and cytoplasm, while it was located in the cytoplasm in almost all the HK2-positive tumor tissues (Fig. 1).

In addition, western blot analysis revealed that MACC1 was positive in 20/24 (83.3\%) of the HCC tissues compared with 11/24 (54.2\%) in the adjacent liver tissues, while HK2 was positive in 21/24 (87.5\%) and 13/24 (65.0\%; $\mathrm{P}<0.05)$, respectively (Fig. 1K). The expression levels of the two proteins were significantly higher in the HCC tissues compared with the adjacent liver tissues.

Notably, the IHC data demonstrated that the expression of MACC1 was significantly associated with tumor staining for HK2 (odds ratio 3.89; 95\% confidence interval $(\mathrm{CI})=1.36-11.18$; Spearman's correlation $=0.569 ; \mathrm{P}=0.009)$, as shown in Fig. 1). This suggested that overexpression of the MACC1 protein in HCC tissues occurred with an increase in HK2 protein.

Correlation between the protein expression levels of MACCl and $H K 2$ and the clinical characteristics of HCC. Following analysis of the association between the expression levels of MACC1 and HK2 in HCC tissues and clinicopathological characteristics (Table I), it was revealed that positive MACC1 expression was significantly associated with large tumor size $(\mathrm{r}=0.332$; $\mathrm{P}=0.004)$, a high Edmondson-Steiner classification $(\mathrm{r}=0.269$; $\mathrm{P}=0.016)$ and an advanced tumor-mode-metastasis (TNM) stage $(r=0.254 ; \mathrm{P}=0.023)$. In addition, positive HK2 expression was significantly correlated with high Edmonson-Steiner classification ( $\mathrm{r}=0.382 ; \mathrm{P}=0.001)$ and advanced TNM stage $(\mathrm{r}=0.373$; $\mathrm{P}=0.001$ ), whereas no correlation was identified between other characteristics. These clinical data suggested that MACC1 and HK2 were closely associated with the process of HCC, and implied advanced HCC progression. 
Effect of tumor MACC1 expression on OS

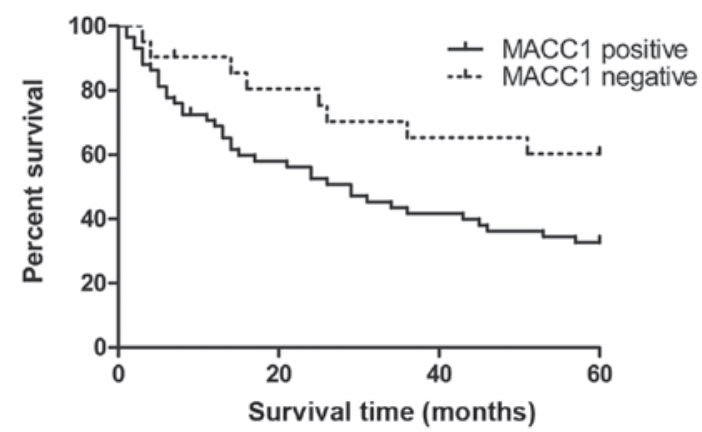

C

OS by MACC1 expression in HK2- tumor cases

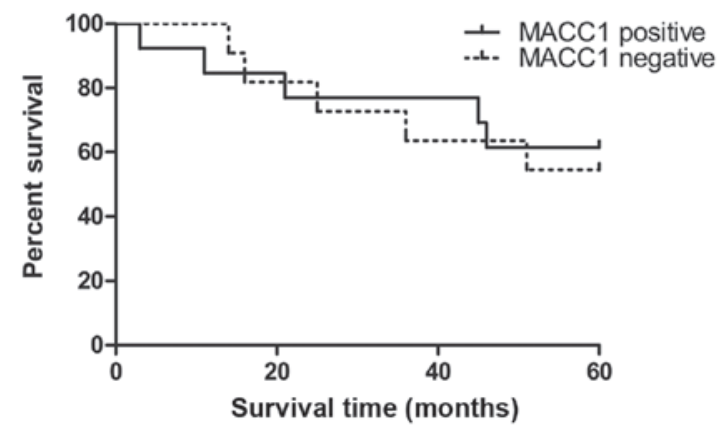

E OS by MACC1 expression in HK2+ tumor cases

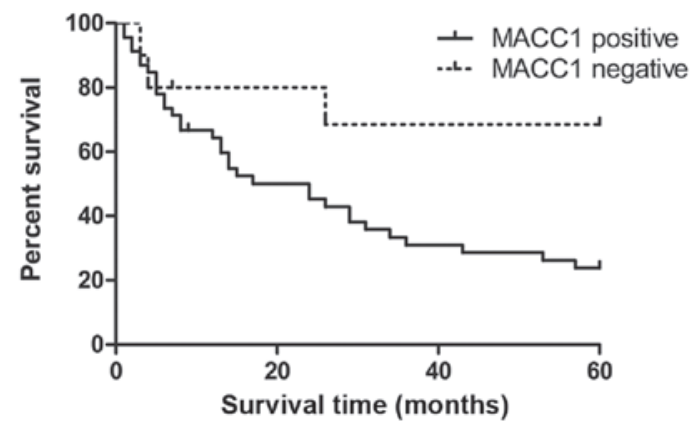

B Effect of tumor HK2 expression on OS

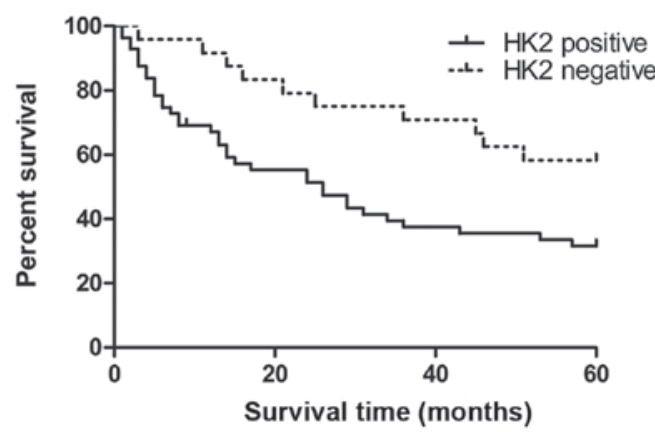

D

OS by HK2 expression in MACC1-tumor cases

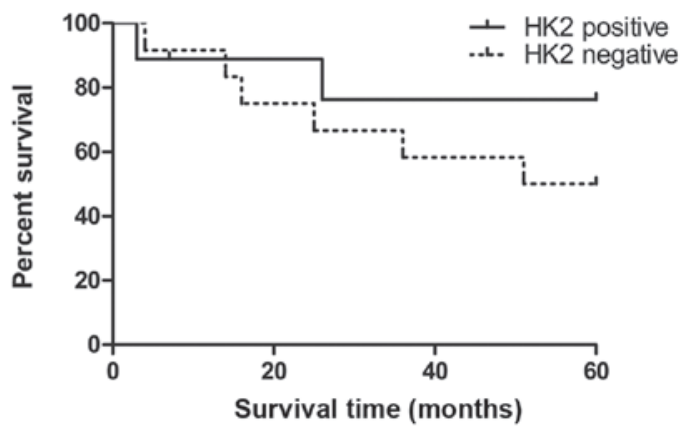

$\mathbf{F}$

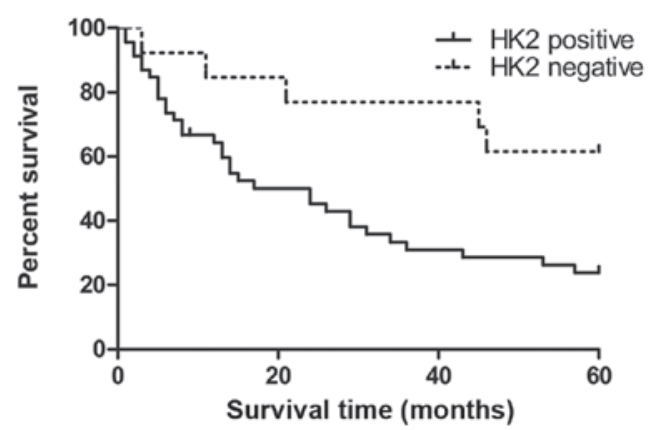

Figure 2. Rates of OS in patients with different expression levels of MACC1 and HK2. Solid lines indicate immunohistochemistry-positive samples while dotted lines indicate immunohistochemistry-negative samples. (A) OS was significantly lower for patients with MACC1-positive tumors compared with patients with MACC1-negative tumors (Log-Rank test $\mathrm{P}=0.032$ ). (B) OS was significantly lower for patients with HK2-positive tumors compared with patients with HK2-negative tumors (Log-Rank test $\mathrm{P}=0.015$ ). (C) Among patients with HK2-negative tumors, OS did not differ significantly on the basis of MACC1 expression (Log-Rank test $\mathrm{P}=0.798$ ). (D) Among patients with MACC1-negative tumors, OS did not differ significantly on the basis of HK2 expression (Log-Rank test $\mathrm{P}=0.308$ ). (E) Among patients with HK2-positive tumors, OS was significantly lower in patients whose tumors were also MACC1-positive (Log-Rank test $\mathrm{P}=0.039$ ). (F) Among patients with MACC1-positive tumors, OS was significantly lower in patients whose tumors were also HK2-positive (Log-Rank test $\mathrm{P}=0.016$ ). OS, overall survival; HK2, hexokinase-2; MACC1, metastasis-associated in colon cancer-1.

A

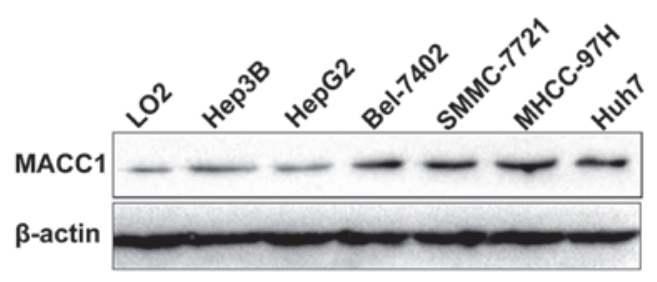

B

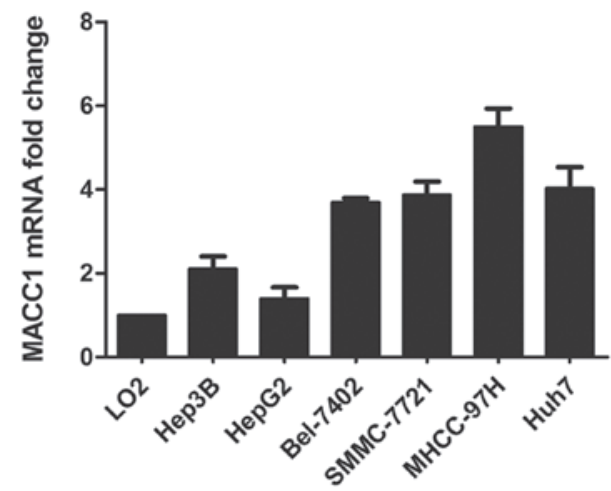

Liver cell lines

Figure 3. Different (A) MACC1 protein and (B) mRNA expression levels in different liver cancer cell lines. Values are expressed as the mean \pm standard error of the mean. MACC1, metastasis-associated in colon cancer-1 gene; HK2, hexokinase-2. 


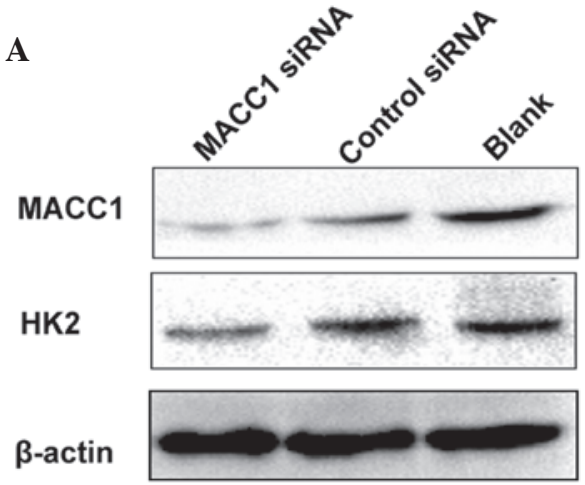

B

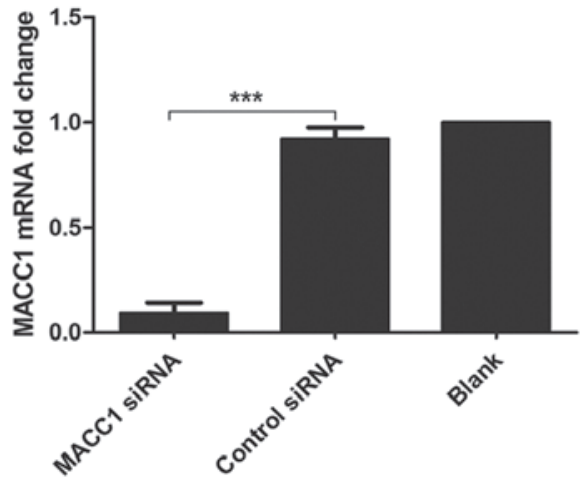

C

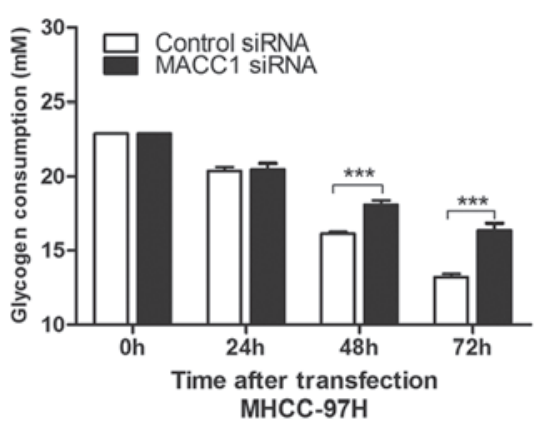

D

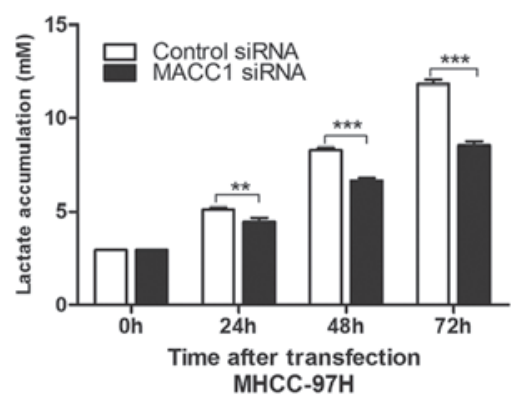

E

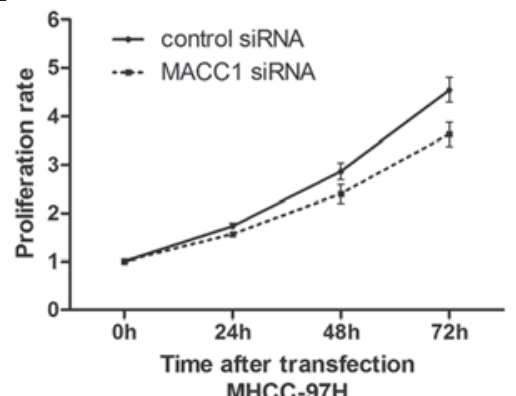

Figure 4. Knockdown of MACC1 inhibits glucose metabolism and reduces the proliferation of MHCC-97H cells. (A and B) expression levels of MACC1 and HK2 in the MHCC-97H cells were significantly decreased following knockdown of MACC1 in western blotting and reverse transcription-quantitative polymerase chain reaction analyses. ( $\left.{ }^{* * * *} \mathrm{P}<0.001\right)$. (C-E) Glucose metabolism and proliferation of the MHCC-97H cells were markedly suppressed by knockdown of MACC1, analyzed using metabolic and MTT assays $\left({ }^{* * *} \mathrm{P}<0.001\right)$. Values are expressed as the mean \pm standard error of the mean. siRNA, small interfering RNA; HK2, hexokinase-2; MACC1, metastasis-associated in colon cancer-1; Blank, untransfected MHCC-97H cells.

Association between survival rates and the tumor expression of MACCl and HK2. Follow-up information was obtained from the $80 \mathrm{HCC}$ cases. The median survival rate of 80 patients was 30 months (range 0-60 months). As compared by a Kaplan-Meier survival curve, MACC1-positive expression was significantly associated with a lower overall survival (OS) rate, with the median survival rate of 29 months in the $59 \mathrm{HCC}$ patients compared with 60 months in the $21 \mathrm{HCC}$ patients with MACC1-negative expression (Log-rank $\mathrm{P}=0.032$; hazard ratio $(\mathrm{HR})=1.99 ; 95 \% \mathrm{CI}=1.06-3.72)$, as shown in Fig. 2A. In addition, HK2-positive expression was also significantly associated with a lower OS rate, with a median survival rate of 26 months in the $56 \mathrm{HCC}$ patients compared with 56 months in the $24 \mathrm{HCC}$ patients with HK2-negative expression (Log-rank $\mathrm{P}=0.015 ; \mathrm{HR}=2.11 ; 95 \% \mathrm{CI}=1.55-3.84)$, as shown in Fig. 2B.

In addition, among the $56 \mathrm{HCC}$ patients with $\mathrm{HK} 2$-positive expression, a lower OS rate was identified in $\mathrm{HCC}$ patients whose samples were also MACC1-positive compared with MACC1-negative tumor samples (Log-rank $\mathrm{P}=0.039$, $\mathrm{HR}=2.31$, 95\% CI=1.04-5.14; Fig. 2C). However, among the $24 \mathrm{HCC}$ patients with HK2-negative expression, no significant change in mortality rate was observed if the patients had MACC1-positive expression (Log-rank P=0.798; Fig. 2E). By contrast, among the 59 HCC patients with MACC1-positive expression, a significantly decreased survival rate was observed in the HCC patients whose samples were also HK2-positive compared with HK2-negative tumor samples (Log-rank $\mathrm{P}=0.016 ; \mathrm{HR}=2.37 ; 95 \% \mathrm{CI}=1.17-4.78$; Fig. 2D). However, among the $21 \mathrm{HCC}$ patients with MACC1-negative expression, no significant change in mortality rate was observed if the patients had HK2 positive expression ( Log-rank $\mathrm{P}=0.308$; Fig. 2F). These data demonstrated that upregulation of the protein expression levels of MACC1 and HK2 led to a poor prognosis in HCC.

Differential protein and $m R N A$ expression levels of MACCI in HCC cell lines. Subsequently, western blot analysis and RT-qPCR were performed in six HCC cell lines (HepG2, Hep3B, SMMC-7721, Bel-7402, Huh7 and MHCC-97H) and a non-transformed human liver cell line (LO2), each of which had different biological characteristics. Among these HCC cell lines, HepG2 expressed the lowest level of MACC1 protein and mRNA, while MHCC-97H expressed a relatively high level of MACC1 protein and mRNA (Fig. 3).

Knockdown of MACC1 in MHCC-97H cells suppresses cell glucose metabolism and reduces proliferation. To further investigate the function of MACC1 in glucose metabolism in cancer, the expression of MACC1 was silenced in MHCC-97H cells by transfection with MACC1 siRNA. As shown in Fig. 4A and B, the protein expression levels of MACC1 and HK2 were significantly decreased following transfection with MACC1 siRNA. The determination of the glycogen concentration of the culture medium revealed that the knockdown of MACC1 markedly suppressed the glucose catabolism of MHCC-97H cells (48 and $72 \mathrm{~h}$ after transfection; $\mathrm{P}<0.001$, respectively; Fig. 4C). Simultaneously, decreased lactate production also confirmed the inhibition of glucose catabolism 

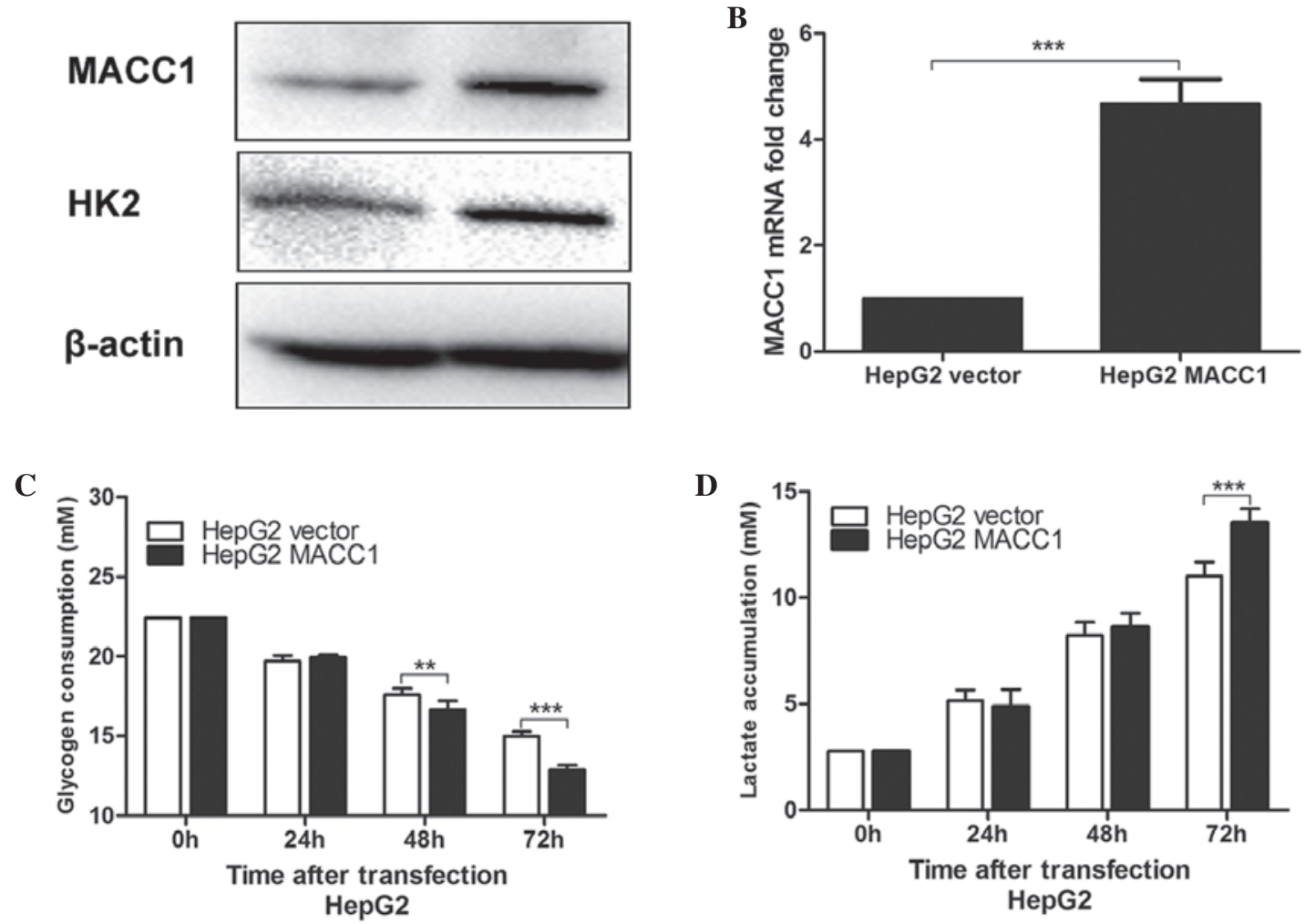

D
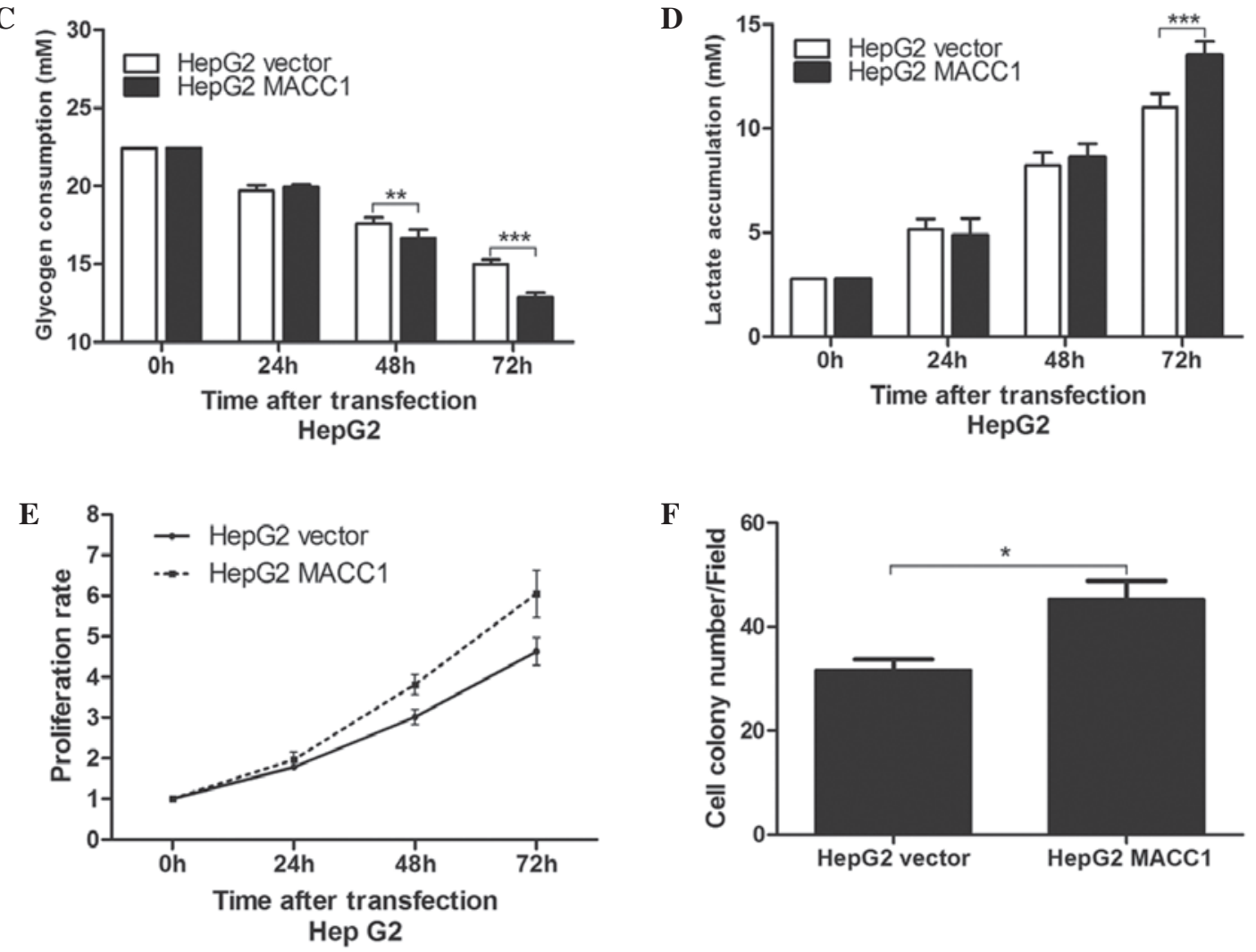

$\mathbf{F}$

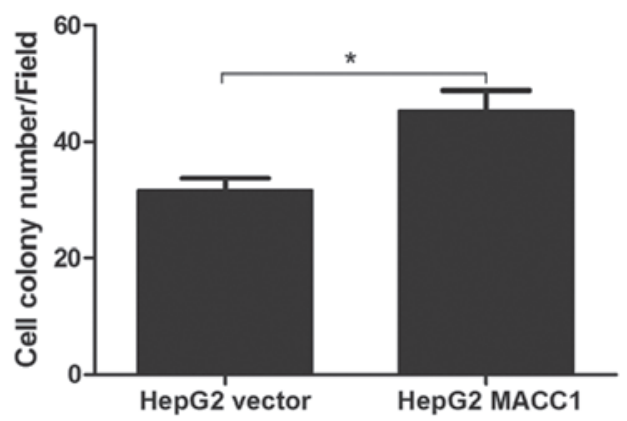

Figure 5. Overexpression of MACC1 enhances glucose metabolism and promotes proliferation of HepG2 cells. (A and B) expression of MACC1 and HK2 in the HepG2 cells were significantly increased by overexpression of MACC1 in western blotting and reverse transcription-quantitative polymerase chain reaction. (C and D) Glucose metabolism of HepG2 cells was markedly activated by overexpression of MACC1 by Metabolic assay. (E and F) Proliferation of HepG2 cells was significantly promoted by overexpression of MACC1 in the MTT and colony formation assay ("P $<0.05$ and $\left.{ }^{* * *} \mathrm{P}<0.001\right)$. Values are expressed as the mean \pm standard error of the mean. HK2, hexokinase-2; MACC1, metastasis-associated in colon cancer-1.

(48 and $72 \mathrm{~h}$ after transfection; P<0.001, respectively; Fig. 4D). The proliferation of MHCC-97H cells was evaluated with or without MACC1 siRNA transfection using an MTT assay. The MTT activity of the MHCC-97H cells transfected with MACC1 siRNA was markedly decreased compared with the control cells (48 and $72 \mathrm{~h}$ after transfection; $\mathrm{P}<0.001$, respectively), implying decreased proliferation rates (Fig. 4E). These data suggested that MACC1 promoted glucose metabolism and proliferation.

Overexpression of MACC1 in HepG2 cells promotes cell glucose metabolism and increases replication. To confirm the observed findings, the protein and mRNA expression of
MACC1 in HepG2 cells was increased through stable transfection with a MACC1-expressing plasmid, and the protein expression of HK2 increased accordingly (Fig. 5A and B). Subsequently, it was identified that the glycogen consumption of the HepG2 cells stably transfected with the MACC1 expressing plasmid (HepG2 MACC1 cells) was significantly higher compared with that of HepG2 cells transfected with the vector plasmid (HepG2 vector cells) 48 and $72 \mathrm{~h}$ after planting $(\mathrm{P}<0.01$; Fig. 5C), with a similar trend in lactate accumulation (72 h after planting; $\mathrm{P}<0.01$; Fig. 5D). The proliferation of the transfected HepG2 was subsequently evaluated using MTT and colony formation assays. The proliferation rate of the HepG2 MACC 1 cells was significantly increased compared with 
the HepG2 vector cells via the MTT assay 48 and $72 \mathrm{~h}$ after planting $(\mathrm{P}<0.01$; Fig. 5E). Furthermore, the colony forming ability of the HepG2 MACC1 cells was significantly enhanced compared with the HepG2 vector cell, as expected $(\mathrm{P}<0.05$; Fig. 5F). These results further clarified that the overexpression of MACC1 promoted proliferation, partly through enhanced glucose metabolism.

\section{Discussion}

The present study introduced a noteworthy finding, confirmed by a number of in vitro assays, that the expression of MACC1 was significantly upregulated in HCC tissues and was accompanied by the increased protein expression of HK2. Clinical data and survival curve analysis revealed that increased protein expression levels of MACC1 and HK2 indicated a poor prognosis in HCC. In addition, knockdown of MACC1 in the MHCC-97H cells led to a reduced rate of proliferation via a reduction in glucose metabolism by the decreased protein expression of HK2. Furthermore, overexpression of MACC1 in the HepG2 cells induced the opposite result, providing confirmation. It was hypothesized that MACC1 leads to a poor prognosis in $\mathrm{HCC}$, partly through promoting proliferation via enhanced glucose metabolism.

$\mathrm{HCC}$ is one of the most common types of cancer worldwide and has a poor prognosis. Tumor resection at an early stage remains the most effective treatment for $\mathrm{HCC}$, however, the lack of early diagnosis has resulted in numerous patients with HCC being unable to undergo resection. In the present study, the role of MACC1 and HK2, from clinical data, and the prognosis of HCC, from immunohistochemical, immunoblotting and survival curve analysis, were investigated and the protein expression levels of MACC1 and HK2 were markedly upregulated in HCC tissue and associated with high Edmondson-Steiner classification, advanced TNM stage and a poor prognosis in patients with $\mathrm{HCC}$. These data indicated the potential development of MACC1 and HK2 as clinical prognostic biomarkers in the future.

As an oncogene, MACC1 contributes to neoplastic growth, invasion and metastasis through the activation of HGF/MET signaling in several types of tumor $(5,9)$. Previous studies have revealed that increased protein expression levels of MACC1 and $\mathrm{HK} 2$ are significantly associated with a poor prognosis in HCC patients $(17,23,24)$. However, the mechanism by which MACC1 and HK2 lead to a poor prognosis remains to be elucidated. The PI3K/Akt signaling pathway, downstream of $\mathrm{HGF} / \mathrm{MET}$, is known as one of the basic elements in the development of several types of tumor, involved in tumor cell growth, differentiation and apoptosis $(25,26)$, and previous studies have demonstrated that the PI3K/Akt signaling pathway is also involved in glucose metabolism in tumors, through increased HK2 protein expression and phosphorylation (19,27-29). Therefore, it was hypothesized that MACC1 induced the tumor growth via promoting tumor glucose metabolism and led to a poor prognosis in patients with HCC. In the present study, the correlation between the protein expression levels of MACC1 and HK2 was initially confirmed using immunohistochemical and western blot analysis in HCC tissue. Subsequently, it was identified that knockdown of MACC1 in MHCC-97H cells decreased the protein expression of HK2, which also inhibited tumor glucose metabolism and reduced proliferation, and may improve prognosis. Subsequent overexpression investigations confirmed these findings. These data suggested that MACC1 induced tumor glucose metabolism by increasing the protein expression of HK2 and caused rapid proliferation, which may lead to poor survival rates in patients with HCC. Although MACC1 was found to enhance the expression of HK2 in the MHCC-97H and HepG2 cells, whether MACC1 regulates the transcription of $\mathrm{HK} 2$ directly remains to be elucidated. The present study aimed to examine the MACC1-binding site of the HK2 promoter by performing out an electrophoretic mobility shift assay, however no positive results were obtained, which indicated that MACC1 may upregulate the expression of HK2 expression in an indirect manner.

In conclusion, the present study demonstrated that aberrantly increased expression levels of MACC1 and HK2 in $\mathrm{HCC}$ tissues were associated with a high Edmondson-Steiner classification and advanced TNM stage, and led to a poor prognosis in HCC patients. In addition, MACC1 induced tumor glucose metabolism by upregulating HK2 indirectly and causing high levels of proliferation, which may be, in part the reason for the poor prognosis in HCC. MACC1 and HK2 may become potential clinical prognosis factors and targets for the treatment of $\mathrm{HCC}$, however, further investigation is required to clarify the specific mechanism by which $\mathrm{MACC} 1$ promotes the progression of HCC.

\section{Acknowledgements}

The present study was supported by grants from the National Natural Scientific Foundation of China (grant no. 81071897 to Yingmin Yao) and the Research Fund for the Doctoral Program of High Education of China from the Ministry of Education (grant no. 20120201120090 to Xin Zheng).

\section{References}

1. Parkin DM, Bray F, Ferlay J and Pisani P: Global cancer statistics, 2002. CA Cancer J Clin 5: 74-108, 2005.

2. Blum HE. Treatment of hepatocellular carcinoma. Best Pract Res Clin Gastroenterol 19: 129-145, 2005.

3. Shimokawa H, Uramoto H, Onitsuka T, et al: Overexpression of mACC1 mRNA in lung adenocarcinoma is associated with postoperative recurrence. J Thorac Cardiovasc Surg 4: 895-898, 2011

4. Shirahata A, Sakata M, Kitamura Y, et al: MACC1 as a marker for peritoneal-disseminated gastric carcinoma. Anticancer Res 30: 3441-3444, 2010.

5. Shirahata A, Shinmura K, Kitamura Y, et al: MACC1 as a marker for advanced colorectal carcinoma. Anticancer Res 30: 2689-2692, 2010.

6. Arlta F and Stein U: Colon cancer metastasis: MACC1 and Met as metastatic pacemakers. Int J Biochem Cell Biol 41: 2356-2359, 2009.

7. Huang Y, Zhang H, Cai J, et al: Overexpression of MACC1 and Its significance in human breast cancer progression. Cell Biosci 1: 16, 2013.

8. Gao J, Ding FH, Liu QG and Yao YM: Knockdown of MACC1 expression suppressed hepatocellular carcinoma cell migration and invasion and inhibited expression of MMP2 and MMP9. Mol Cell Biochem 376: 21-32, 2012.

9. Stein U, Walther W, Arlt F, et al: MACC1, a newly identified key regulator of HGF-MET signaling, predicts colon cancer metastasis. Nat Med 15: 59-67, 2009.

10. Qiu J, Huang P, Liu Q, et al: Identification of MACC1 as a novel prognostic marker in hepatocellular carcinoma. J Transl Med 9: 166-176, 2011.

11. Wallace DC: Mitochondria and cancer: Warburg addressed. Cold Spring Harb Symp on Quantit Biol 70: 363-374, 2005. 
12. Mathupala SP, Rempel A and Pedersen PL: Aberrant glycolytic metabolism of cancer cells: a remarkable coordination of genetic, transcriptional, post-translational, and mutational events that lead to a critical role for type II hexokinase. J Bioenerg Biomembr 29: 339-343, 1997.

13. Bustamante E and Pedersen PL: High aerobic glycolysis of rat hepatoma cells in culture: role of mitochondrial hexokinase. Proc Natl Acad Sci USA 74: 3735-3739, 1977.

14. Bustamante E, Morris HP and Pedersen PL: Energy metabolism of tumor cells: requirement for a form of hexokinase with a propensity for mitochondrial binding. J Biol Chem 256: 8699-8704, 1981.

15. Pastorino JG and Hoek JB: Hexokinase II: the integration of energy metabolism and control of apoptosis. Curr Med Chem 10: $1535-1551,2003$

16. Sattler M and Salgia R: c-Met and hepatocyte growth factor: potential as novel targets in cancer therapy. Curr Oncol Rep 9: 102-108, 2007

17. Lang AH, Geller-Rhomberg S, Winder T, et al: A common variant of the MACC1 gene is significantly associated with overall survival in colorectal cancer patients. BMC Cancer 12: 20, 2012.

18. Ahn KJ, Hwang HS, Park JH, et al: Evaluation of the role of hexokinase type II in cellular proliferation and apoptosis using human hepatocellular carcinoma cell lines. J Nucl Med 50: 1525-1532, 2009.

19. Roberts DJ, Tan-Sah VP, Smith JM and Miyamoto S: Akt phosphorylates HK-II at Thr473 and increases mitochondrial HK-II association to protect cardiomyocytes. J Biol Chem 288 23798-23806, 2013

20. Albers I, Hartmann H, Bircher J and Creutzfeldt W: Superiority of the Child-Pugh classification to quantitative liver function tests for assessing prognosis of liver cirrhosis. Scand J Gastroenterol 24: 269-276, 1989.
21. Shephard DA: The 1975 Declaration of Helsinki and consent. Can Med Assoc J 115: 1191-1192, 1976.

22. Liu F, Yang T, Wang B, et al: Resistin induces insulin resistance, but does not affect glucose output in rat-derived hepatocytes. Acta Pharmacol Sin 29: 98-104, 2008.

23. Xie C, Wu J, Yun J, et al: MACC1 as a prognostic biomarker for early-stage and AFP-normal hepatocellular carcinoma. PLoS One 8: e64235, 2013.

24. Ge SH, Wu XJ, Wang XH, et al: Over-expression of metastasis-associated in colon cancer-1 (MACC1) associated with better prognosis of gastric cancer patients. Chin J Cancer Res 23: 153-159, 2011

25. Gottlob K, Majewski N, Kennedy S, Kandel E, Robey RB and Hay N: Inhibition of early apoptotic events by Akt/PKB is dependent on the first committed step of glycolysis and mitochondrial hexokinase. Genes Dev 15: 1406-1418, 2001.

26. Bijur GN and Jope RS: Rapid accumulation of Akt in mitochondria following phosphatidylinositol 3-kinase activation. J Neurochem 87: 1427-1435, 2003.

27. Neary CL and Pastorino JG: Akt inhibition promotes hexokinase 2 redistribution and glucose uptake in cancer cells. J Cell Physiol 228: 1943-1948, 2013.

28. Zhu Y, Pereira RO, O'Neill BT, et al: Cardiac PI3K-Akt impairs insulin-stimulated glucose uptake independent of mTORC1 and GLUT4 translocation. Mol Endocrinol 27 172-184, 2013.

29. Gwak GY, Yoon JH, Kim Km, Lee HS, Chung JW, Gores GJ: Gores. Hypoxia stimulates proliferation of human hepatoma cells through the induction of hexokinase II expression. J Hepatol 42: $358-364,2005$. 